\title{
Spectroscopic Evaluation of the Atomic Size
}

\author{
Nazmul Islam and Dulal C. Ghosh* \\ Department of Chemistry, University of Kalyani, Kalyani-741235, India
}

\begin{abstract}
A new algorithm for evaluating the atomic size is suggested by entailing the atomic spectroscopic data-the wave number. The basic tenet of the present method is (i) to convert a multi-electron atom system to a hydrogenic atom to invoke the Bohr model for the mechanism of electron transition, and (ii) to use the experimental atomic spectroscopic data of multi electron systems to determine the atomic radii. The estimated set of size data appears to satisfy the entire 'sine qua non' of sizes of atoms of the periodic table. Relativistic effect appears to have been significantly included in the suggested algorithm for evaluating the atomic radii. The express periodicity of periods and groups of periodic table exhibited by the computed atomic radii, $\mathrm{d}$ and $\mathrm{f}$ block contraction and the manifestation of the relativistic effect in the sizes of lanthanoids and actinoids etc speak volume of the efficacy of the present method in computing atomic size. Furthermore, as a validity test, the size data evaluated in the present work have been exploited to calculate some physical descriptors of the real world like equilibrium inter nuclear distances of a good number of hetero nuclear diatomics. We have noted the surprisingly close agreement between the theoretical and the experimental equilibrium inter-nuclear distances.
\end{abstract}

Keywords: Atomic radii, Wave number, Global hardness, Bohr model, Relativistic Effect, Inter nuclear distances of hetero nuclear diatomic molecules.

\section{INTRODUCTION}

According to Feynman [1] the atom is a basic unit of matter that consists of a dense, central nucleus surrounded by a cloud of negatively charged electrons. In other words, all things are made of atoms. Atom has a size. It is opined that if the electron mass would have been different, as a consequence, the sizes of atoms and sizes of things around would have been different-so important is the size of the atom. Scientists engaged in explaining the universe in terms of Higgs field and Higgs boson, believe that the sizes of the molecule and in turn the size of the atoms are determined by the paths of the electron orbiting the nucleus. The size of those orbits, however, is determined by the mass of the electron. So understanding the mass of the electron is essential to understanding the size and dimension of everything around us. But the more the science is advancing the exact size of the atom is becoming queerer and queerer.

Till date, it has not been possible to isolate a single atom and to determine its size. Since the size of the atom is not an experimental quantity, no quantum mechanical operator can be suggested and the possibility of its quantum mechanical measurement is ruled out according to the rules of quantum mechanics. Quantum mechanics, also known as quantum physics or quantum theory, is a branch of physics providing a mathematical description of the electronic structure and properties of atoms, molecules and crystalline bodies. It is known from quantum mechanics that the probability of finding an electron anywhere in space is finite and gradually

*Address correspondence to this author at the Department of Chemistry, University of Kalyani, Kalyani-741235, India; Tel: +91-33-2582 8282; Fax: +91-33-2582 8282; E-mail: dcghosh1@ rediffmail.com diminishes as the radial distance increases and becomes zero at infinity. This implies that the atoms, ions and molecules do not have any rigid shape or size and the radius of an atom is infinity. But large body of physical facts and experience force us to believe that atoms and ions do have a finite size for all practical purposes. The legend 'atomic radius' is an important size descriptor of atoms required in correlating, predicting and modeling many physico-chemical properties of atoms, molecules and structural aspects of condensed matter. The right size of ions and atoms are of paramount importance in modeling and understanding bio-chemical processes. Furthermore, atomic radii are associated with physico-chemical properties such as electronegativity [2], global hardness [3, 4], ionization energy [5-10], polarizability [3], diamagnetic susceptibility [3], capacitance [11], electrophilicity index [12] etc. Thus the concept of 'atomic radius' is the edifice of the conceptual construct of physics and chemistry.

We are habituated in thinking and modeling in terms of many things that do not follow from quantum mechanics. The atomic radius, the hardness and the electronegativity are important conceptual constructs of chemistry. Without the concept and operational significance of radius, hardness and electronegativity, chemistry and many aspects of condensed matter physics become chaotic and the long established unique order in chemico-physical world would be disturbed. But fact remains that the radius, electronegativity and chemical hardness are conundrums and objects of purely intellectual intuition and are not things of real world. They occur in mind like the unicorn of mythical saga [13]. According Kant, these are noumenon - objects knowable by the mind or intellect but not by the senses. These quantities are qualitative concept per se and have to be evaluated qualitatively goaded 
by physical and chemical experience. Hence, before any algorithm of computing the radius, hardness and the electronegativity is developed, the reification of abstract concepts regarding the above descriptors into things of the real world is absolutely necessary.

Thus, in order to assign some number to each of these abstract concepts like the atomic radius, the electronegativity and the hardness, it is required that these should be reified [14] in terms of the physico-chemical behavior of such conundrums goaded by the quantum mechanical principles and then, some mathematical algorithm will be developed. We must keep in mind the commonality between such descriptors as are under discussion. All these descriptors are periodic in nature and their mutual relationship is goaded by the periodic law. It is pertinent to mention here that the periodic table has strong chemical organizing power and it is not surprising that correlations can be found between seemingly unrelated quantities because of their periodicity. The atomic radius, electronegativity and hardness are all periodic and hence they should be mutually related. The shell structure and screened nuclear charge, the internal constitution of atom control the size of atom, electronegativity and hardness. The screened nuclear charge increases horizontally along a period. Since the screened nuclear charge is primarily controlling the atomic and ionic sizes, the size should decrease and electronegativity and hardness should increase monotonically along the horizontal row [15]. This further suggests that electronegativity, hardness and the atomic radius are intimately connected to each other because of their commonality in the origin and development.

\section{THE RELATION BETWEEN THE ATOMIC RADIUS AND THE IONIZATION POTENTIAL}

The history of determination of atomic size is quite old and a good number of theoretical algorithms, of both semiempirical and non-empirical nature, are suggested to evaluate the sizes of atoms and ions [3,5-10,16-36]. The relation between the atomic radius (volume) and the ionization potential has been investigated by a number of doyens of science from as early as 1920 [5-10]. In 1921, Eve [8] pointed out that the ionization energy of an atom is inversely proportional as the radius. Saha [6] took an extensive study to find the relationship between ionization potential and atomic radius and relied upon the suggestion of Bose [7] that the field of force exerted by the central nucleus upon the valence electrons is important in determining the size of the atoms. However, the suggestion of Bose [7] for many electron atom is the concept of effective nuclear charge of the present day science. In 1996, Tamura [9] pointed out that the first ionization energy can be predicted from the atomic radius. Recently, Bohórquez et al., [10] has proposed a semi-empirical definition of the radius of an atom in terms of its ionization energy. Since the effective nuclear charge, ionization energy and atomic radii are all periodic in nature, they are correlated with each other. Recently, Ghosh et al., [37] proposed a model of computing atomic radii through the conjoint action of the effective nuclear charge and the ionization energy.

Although a number of reports of calculating the atomic radius entailing there are the ionization energy [5-10, 37], all are empirical in nature and it seems that these works use the proportionality relationship between atomic radius and the ionization energy. But the necessary algorithm relating ionization energy and atomic radius from the fundamental standpoint is still at large.

The relativity creeps in the shell structure and the size of the atom, any calculation of the atomic size must consider the relativistic effect, at least, for heavier elements. So far, there is no report of evaluation of atomic size in terms of spectroscopic data of atoms and in the present effort we are suggesting a model of computing atomic size invoking the spectral data of atoms.

In this venture we are exploiting the inter relationship between three descriptors-the global hardness, the ionization energy and the atomic radius, The experimental spectral data must have subsumed the effect of electron correlation and relativity. In the exploration of the relationship between the radius and the ionization energy of the atoms, we have relied upon our new definition of the global hardness of the atoms [38-41].

\section{THE QUANTIFICATION OF THE HYPOTHETICAL CONCEPT OF GLOBAL HARDNESS}

Parr and co-workers, using the Density Functional Theory, (DFT) [42, 43], as basis, placed the qualitative hard-soft acid base, HSAB principle and many other conceptual constructs of chemistry and physics on the plat form of quantum mechanical theory. Given the electron density function $\rho(\mathrm{r})$ in a chemical system and the energy functional $E(\rho)$, the chemical potential, $\mu$ of that system in equilibrium has been defined as the derivative of the energy with respect to the electron density at fixed molecular geometry.

The chemical potential [44], $\mu$, is given by-

$\mu=[\delta \mathrm{E}(\rho) / \delta \rho]_{\mathrm{v}}$

where $\mathrm{v}$ is the external potential acting on an electron due to the presence of nucleus.

The differential definition more appropriate to atomic system is on the basis that for a system of $\mathrm{N}$ electrons with ground state energy $\mathrm{E}[\mathrm{N}, \mathrm{v}]$,

$\mu=[\partial \mathrm{E} / \partial \mathrm{N}]_{\mathrm{v}}$

Following Iczkowski and Margrave [45], Parr et al., [46] defined:

$\chi=-\mu$

or, $\chi=-[\partial \mathrm{E} / \partial \mathrm{N}]_{\mathrm{v}}$

where $\chi$ is the electronegativity

The absolute hardness [47] $\eta$, was defined as

$\eta=1 / 2[\partial \mu \partial \mathrm{N}]_{\mathrm{v}}=1 / 2\left[\left(\partial^{2} \mathrm{E} / \partial \mathrm{N}^{2}\right)\right]_{\mathrm{v}}$

Although rigorous mathematical formulae $[47,48]$ were suggested, the evaluation of hardness in terms of the suggested formulae in Eq. (5) is difficult [49-51]. However, calculus of finite difference approximation was invoked [47] to suggest an approximate and operational formula of hardness as under:

$\eta=1 / 2(\mathrm{I}-\mathrm{A})$

where ' $\mathrm{I}$ ' and 'A' are the first ionization potential and electron affinity of the chemical species. 
We [4] have suggested a new electrostatic definition and derived a new radial dependent formula for computing atomic hardness as follows-

Classically, the energy $\mathrm{E}(\mathrm{N})$ of charging a conducting sphere of radius $r$ with charge $q$ is given by

$\mathrm{E}(\mathrm{N})=\mathrm{q}^{2} / 2 \mathrm{r}$ (in C.G.S. Unit)

$\mathrm{E}(\mathrm{N})=\mathrm{q}^{2} /\left(4 \pi \varepsilon_{0}\right) 2 \mathrm{r}$ (in S.I Unit)

In Eq. (7), $\mathrm{E}(\mathrm{N})$ is in ergs, $\mathrm{q}$ is in electrostatic unit and $\mathrm{r}$ is in $\mathrm{cm}$. Now, for an atom, the change in energy associated with the change in q on removal of an electron (of charge, e), would be the ionization energy, I. Similarly, the energy evolved on addition of an electron with $q$ would be the electron affinity, A.

Hence,

$\mathrm{I}=\mathrm{E}(\mathrm{N}+1)-\mathrm{E}(\mathrm{N})=\left[\left\{(\mathrm{q}+\mathrm{e})^{2} / 2 \mathrm{r}\right\}-\left(\mathrm{q}^{2} / 2 \mathrm{r}\right)\right]$

and

$\mathrm{A}=\mathrm{E}(\mathrm{N})-\mathrm{E}(\mathrm{N}-1)=\left[\left(\mathrm{q}^{2} / 2 \mathrm{r}\right)-\left\{(\mathrm{q}-\mathrm{e})^{2} / 2 \mathrm{r}\right\}\right]$

Now, putting the values of I and A from above into the Eq. (6), we get-

$\eta=(1 / 2)\left[\left\{(\mathrm{q}+\mathrm{e})^{2} / 2 \mathrm{r}\right\}-\left(\mathrm{q}^{2} / 2 \mathrm{r}\right)-\left(\mathrm{q}^{2} / 2 \mathrm{r}\right)+\left\{(\mathrm{q}-\mathrm{e})^{2} / 2 \mathrm{r}\right\}\right]$

or, $\eta=\mathrm{e}^{2} / 2 \mathrm{r}$

where e is the electronic charge in esu and $r$ is the most probable radius of the atom in $\mathrm{cm}$.

Comparing the two equations of computing global hardness of atoms, the Parr Pearson's equation (Eq. 6), and the equation (Eq. 12) derived by us, we get

$(\mathrm{I}-\mathrm{A}) / 2=\mathrm{e}^{2} / 2 \mathrm{r}$

or, $r=\mathrm{e}^{2} /(\mathrm{I}-\mathrm{A})$

Now in most cases of atoms, A is either negligibly small or zero $[14,15,52,53]$, we can simplify the Eq. (14) as

$\mathrm{r}=\mathrm{e}^{2} / \mathrm{I}$

Thus, hereby we introduce a new quantitative relationship between the size and the ionization potential of the atom. We have calculated the size of the atoms of 103 elements of periodic table though the Eqn (15) in terms of the ionization energies of the atoms evaluated through spectroscopic method.

\section{SPECTROSCOPIC METHOD OF DETERMINA- TION OF IONIZATION POTENTIAL AND ATOMIC RADIUS}

We have developed above that the ionization energy can be directly linked to the atomic radius. We have evaluated atomic size through equation (15) without using the ionization energy determined experimentally or evaluated through Hartree-Fock SCF scheme and invoking Koopmans theorem. Rather, we have explored a new route relying upon spectral transitions and spectroscopy. In spectral transitions and spectral data, the ionization energy is subsumed.

Let the transion occurs from higher energy level, $\mathrm{E}_{\mathrm{U}}$ pper to a lower energy level $\mathrm{E}_{\text {Lower }}$ in a hydrogenic atom and also let for this transition, a photon of wave number, $\bar{v}$, is emitted.
From classical Bohr model [5], we write the energy of upper state as

$\mathrm{E}_{\text {Upper }}=-\mathrm{hcR} \mathrm{R}_{\mathrm{H}} / \mathrm{n}^{2}$

where $\mathrm{c}$ is the velocity of light, $\mathrm{R}_{\mathrm{H}}$ is the Rydberg constant and $\mathrm{n}$ is the principal quantum number of the upper energy label.

We know that, from the principle of conservation of energy,

$\Delta \mathrm{E}=\mathrm{E}_{\text {Lower }}-\mathrm{E}_{\text {Upper }}=\mathrm{h} \mathrm{c} \overline{\mathrm{v}}$

Putting the value of $\mathrm{E}_{\mathrm{Upper}}$ and rearranging the equation above, we get-

$\bar{v}=-R_{H} / n^{2}-E_{\text {lower }} / h c$

Since, the ionization energy, I, is given by the definition

$\mathrm{I}=-\mathrm{E}_{\text {lower }}$

It follows that

$\overline{\mathrm{V}}=\mathrm{I} / \mathrm{hc}-\mathrm{R}_{\mathrm{H}} / \mathrm{n}^{2}$

Rearranging Eq.(20), we get

$\mathrm{I}=\mathrm{hc}\left(\overline{\mathrm{v}}+\mathrm{R}_{\mathrm{H}} / \mathrm{n}^{2}\right)$

The Rydberg constant, $\mathrm{R}_{\mathrm{H}}$ has the general form-

$\mathrm{R}_{\mathrm{H}}=2 \pi^{2} \mathrm{Z}^{2} \mathrm{e}^{4} \mathrm{~m} / \mathrm{h}^{3} \mathrm{c}$

where $\mathrm{Z}$ is the actual nuclear charge, e is the electronic charge, $m$ is the mass of the electron.

The basic tenet of the present method is (i) to convert a multi-electron atom system to a hydrogenic atom to invoke the Bohr model for the mechanism of electron transition, and (ii) to use the experimental atomic spectroscopic data of multi electron systems to determine the atomic radii.

The simple way of doing this is to modify the above expression of $\mathrm{R}_{\mathrm{H}}$ by replacing the actual nuclear charge, $\mathrm{Z}$ by the corresponding effective nuclear charge $Z_{\text {eff, of }}$ atom. These have virtually converted a multi-electron atom to hydrogenic atom.

The modified Rydberg constant looks

$\mathrm{R}_{\mathrm{H}}^{\prime}=2 \pi^{2} \mathrm{Z}_{\mathrm{eff}}{ }^{2} \mathrm{e}^{4} \mathrm{~m} / \mathrm{h}^{3} \mathrm{c}$

Using the modified Rydberg constant, $\mathrm{R}_{\mathrm{H}}^{\prime}$ and replacing the principal quantum number, $\mathrm{n}$ by the effective principal quantum number, $\mathrm{n}^{*}$ we rewrite the Eq. (20), as follows-

$\mathrm{I}=\mathrm{hc} \overline{\mathrm{v}}+\left(2 \pi^{2} \mathrm{Z}_{\mathrm{eff}}{ }^{2} \mathrm{e}^{4} \mathrm{~m} / \mathrm{h}^{2} \mathrm{n}^{* 2}\right)$

Putting the value of $\mathrm{I}$ in $\operatorname{Eq}(15)$, a relationship between the wave number and the radius of the atom is obtained as follows-

$r=\left(e^{2} / h c \bar{v}\right)+\left(2 \pi^{2} Z_{\text {eff }}{ }^{2} e^{4} m / h^{2} n^{* 2}\right)$

To compute $r$ in angstrom unit, we rearrange the above equation as-

$r=(7.2 / h c \bar{v})+\left(2 \pi^{2} Z_{e f f}^{2} e^{4} m / h^{2} n^{* 2}\right)$

We have computed the atomic radius of 103 elements of the periodic table using the proposed Eq (26). The appropriate wave number $(v)$ values for different elements are taken from reference [54] and are presented in Table 1. Furthermore, we have used the effective nuclear charge, $Z_{\text {eff }}$ published by 
Table 1. Wave Numbers, Computed Atomic Radii(au), Ghosh et al., radii(au), Desclaux`s radii(au) and Waber and Cromer's Radii(au)

\begin{tabular}{|c|c|c|c|c|c|}
\hline $\mathrm{H}$ & 109679 & 1 & 1 & 1.01 & \\
\hline $\mathrm{Li}$ & 43487 & 2.5 & 3.08 & 3.1 & 3.08 \\
\hline $\mathrm{C}$ & 90820 & 1.19 & 1.23 & 1.22 & 1.23 \\
\hline $\mathrm{N}$ & 117226 & 0.92 & 1.03 & 0.99 & 1.03 \\
\hline $\mathrm{O}$ & 109837 & 0.98 & 0.88 & 0.83 & 0.87 \\
\hline $\mathrm{Mg}$ & 61671 & 1.75 & 3.16 & 2.58 & 3.16 \\
\hline $\mathrm{Al}$ & 48278 & 2.21 & 2.57 & 2.69 & 2.57 \\
\hline $\mathrm{Si}$ & 65748 & 1.64 & 2.17 & 2.18 & 2.17 \\
\hline $\mathrm{P}$ & 84581 & 1.28 & 1.87 & 1.85 & 1.87 \\
\hline$S$ & 83559 & 1.28 & 1.65 & 1.62 & 1.65 \\
\hline $\mathrm{Cl}$ & 104591 & 1.03 & 1.48 & 1.44 & 1.48 \\
\hline $\mathrm{Ar}$ & 127110 & 0.84 & 1.33 & 1.3 & 1.33 \\
\hline $\mathrm{Cr}$ & 54576 & 1.98 & 3.97 & 3.03 & 4.29 \\
\hline $\mathrm{Mn}$ & 59959 & 1.8 & 3.8 & 2.65 & 4.11 \\
\hline $\mathrm{Fe}$ & 63737 & 1.7 & 3.65 & 2.55 & 3.95 \\
\hline Co & 63565 & 1.7 & 3.51 & 2.46 & 3.79 \\
\hline $\mathrm{Ni}$ & 61619 & 1.74 & 3.38 & 2.38 & 3.65 \\
\hline $\mathrm{Cu}$ & 62317 & 1.73 & 3.26 & 2.54 & 3.52 \\
\hline $\mathrm{Zn}$ & 75769 & 1.43 & 3.15 & 2.23 & 3.4 \\
\hline $\mathrm{Ga}$ & 48388 & 2.2 & 2.74 & 2.65 & 2.96 \\
\hline $\mathrm{Ge}$ & 63713 & 1.67 & 2.42 & 2.27 & 2.62 \\
\hline As & 78950 & 1.36 & 2.16 & 2.02 & 2.35 \\
\hline $\mathrm{Se}$ & 78658 & 1.36 & 1.97 & 1.83 & 2.13 \\
\hline $\mathrm{Br}$ & 95285 & 1.12 & 1.8 & 1.69 & 1.95 \\
\hline $\mathrm{Kr}$ & 112915 & 0.95 & 1.66 & 1.57 & 1.79 \\
\hline $\mathrm{Rb}$ & 33691 & 3.24 & 7.27 & 4.65 & 9.09 \\
\hline
\end{tabular}


Table 1. contd....

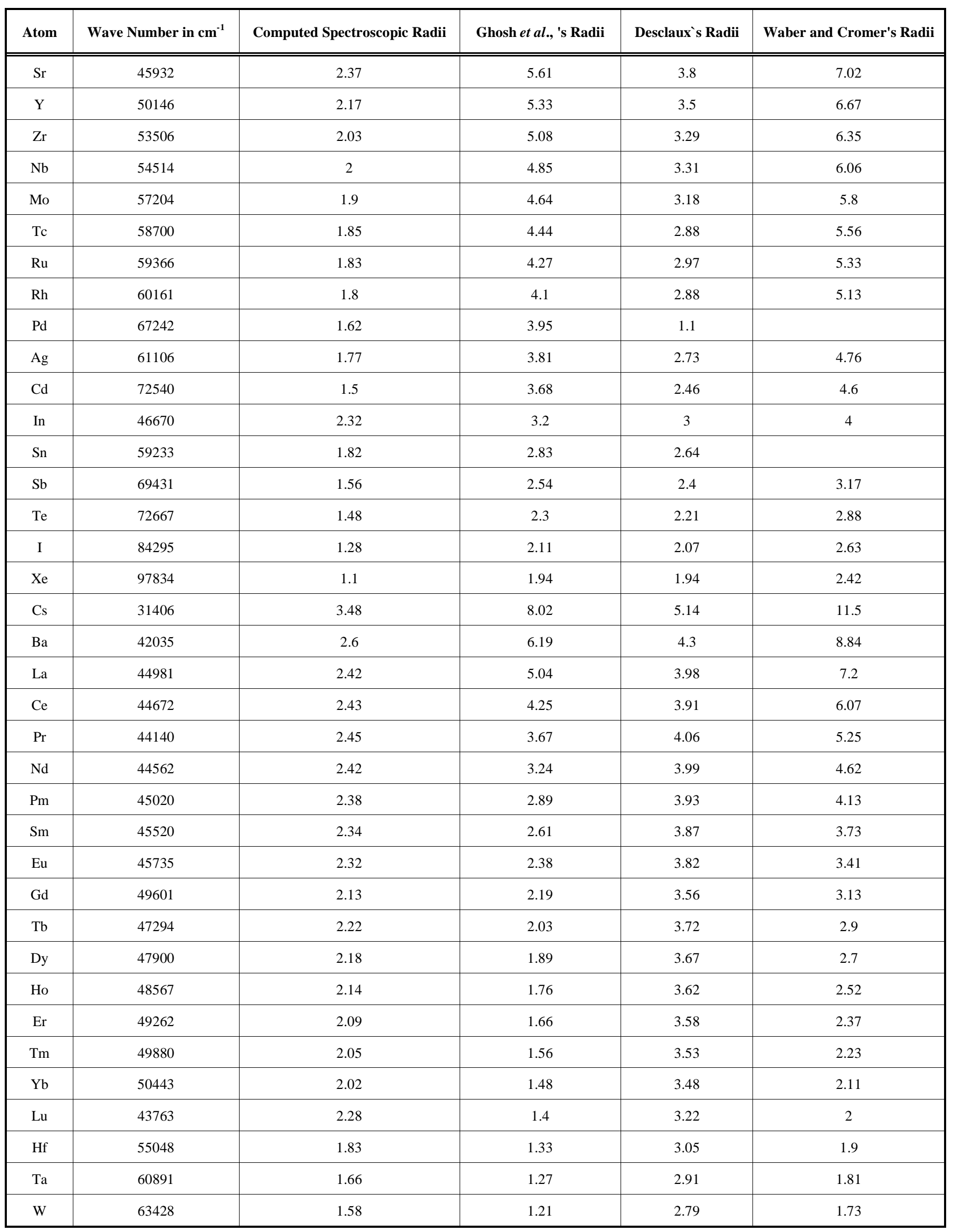


Table 1. contd....

\begin{tabular}{|c|c|c|c|c|c|}
\hline Atom & Wave Number in $\mathbf{c m}^{-1}$ & Computed Spectroscopic Radii & Ghosh et al., 's Radii & Desclaux`s Radii & Waber and Cromer's Radii \\
\hline $\mathrm{Re}$ & 63182 & 1.58 & 1.16 & 2.69 & 1.66 \\
\hline Os & 68079 & 1.46 & 1.11 & 2.6 & 1.59 \\
\hline $\mathrm{Ir}$ & 72324 & 1.37 & 1.07 & 2.51 & 1.55 \\
\hline $\mathrm{Pt}$ & 72297 & 1.4 & 1.03 & 2.53 & 1.47 \\
\hline $\mathrm{Au}$ & 74409 & 1.32 & 0.99 & 2.46 & 1.42 \\
\hline $\mathrm{Hg}$ & 84184 & 1.17 & 0.96 & 2.3 & 5.79 \\
\hline $\mathrm{Tl}$ & 49266 & 2.2 & 3.53 & 3.15 & 5.04 \\
\hline $\mathrm{Pb}$ & 59819 & 1.81 & 3.12 & 2.81 & 4.46 \\
\hline $\mathrm{Bi}$ & 58762 & 1.84 & 2.8 & 2.58 & 4 \\
\hline Po & 67860 & 1.59 & 2.54 & 2.4 & 3.63 \\
\hline At & 77702 & 1.39 & 2.32 & 2.26 & 3.32 \\
\hline $\mathrm{Rn}$ & 86693 & 1.25 & 2.14 & 2.14 & 3.05 \\
\hline $\mathrm{Fr}$ & 32849 & 3.33 & 8.4 & 5 & 13.7 \\
\hline $\mathrm{Ra}$ & 42573 & 2.57 & 6.49 & 4.26 & 10.6 \\
\hline Ac & 41700 & 2.62 & 6.16 & 3.91 & 10 \\
\hline $\mathrm{Th}$ & 50867 & 2.15 & 5.87 & 3.66 & 9.56 \\
\hline $\mathrm{Pa}$ & 47500 & 2.29 & 4.3 & 3.73 & 7 \\
\hline $\mathrm{U}$ & 49958 & 2.17 & 3.74 & 3.66 & 6.08 \\
\hline $\mathrm{Np}$ & 50536 & 2.14 & 3.3 & 3.59 & 5.37 \\
\hline $\mathrm{Pu}$ & 48603 & 2.21 & 2.74 & 3.71 & 4.46 \\
\hline $\mathrm{Am}$ & 48182 & 2.23 & 2.44 & 3.66 & 4.07 \\
\hline $\mathrm{Cm}$ & 48324 & 2.22 & 2.45 & 3.41 & 3.99 \\
\hline $\mathrm{Bk}$ & 49989 & 2.13 & 2.13 & 3.55 & 3.46 \\
\hline $\mathrm{Cf}$ & 50665 & 2.09 & 1.98 & 3.5 & 3.22 \\
\hline Es & 51800 & 2.04 & 1.85 & 3.45 & 3.01 \\
\hline Fm & 52392 & 2.01 & 1.74 & 3.99 & 2.83 \\
\hline Md & 53037 & 1.97 & 1.64 & 3.35 & 2.66 \\
\hline No & 53602 & 1.94 & 1.55 & 3.31 & 2.52 \\
\hline $\mathrm{Lr}$ & 37078 & 2.74 & 1.53 & 3.05 & \\
\hline
\end{tabular}

Ghosh and Biswas [3] and the effective principal quantum number, $n^{*}$ for $n=1$ to 6 published by Slater [17]. In case of $\mathrm{n}=7$, we have used the $\mathrm{n}^{*}$ value suggested by Ghosh and Biswas [3].

The wave numbers and the evaluated atomic radii (au) of 103 elements of the periodic table are presented in the Table 1. For a comparative study, the size data published by Ghosh et al., [36], Waber and Cromer [21], and Desclaux [29] are also tabulated vis-a-vis in the Table 1. In order to explore the periodic behaviour, the evaluated size data is plotted as function of atomic number in Fig. (1).
The evaluated radii of $3 \mathrm{~d}$ block elements, lanthanoids and actinoids vis-à-vis the radii data of such elements published by Desclaux[29] are plotted in the Figs. (2-4) respectively.

As we have already pointed out that there is no experimental or theoretical bench-mark of atomic radii to perform the validity test of any scale of evaluation of absolute radii, we have applied our computed radii to correlate the major chemico-physical behaviour of atoms and to evaluate a real world descriptor like inter-nuclear bond distances of nine sets of hetero nuclear diatomic molecules with diverse physico-chemical behaviour. The diatomic molecules have gained 


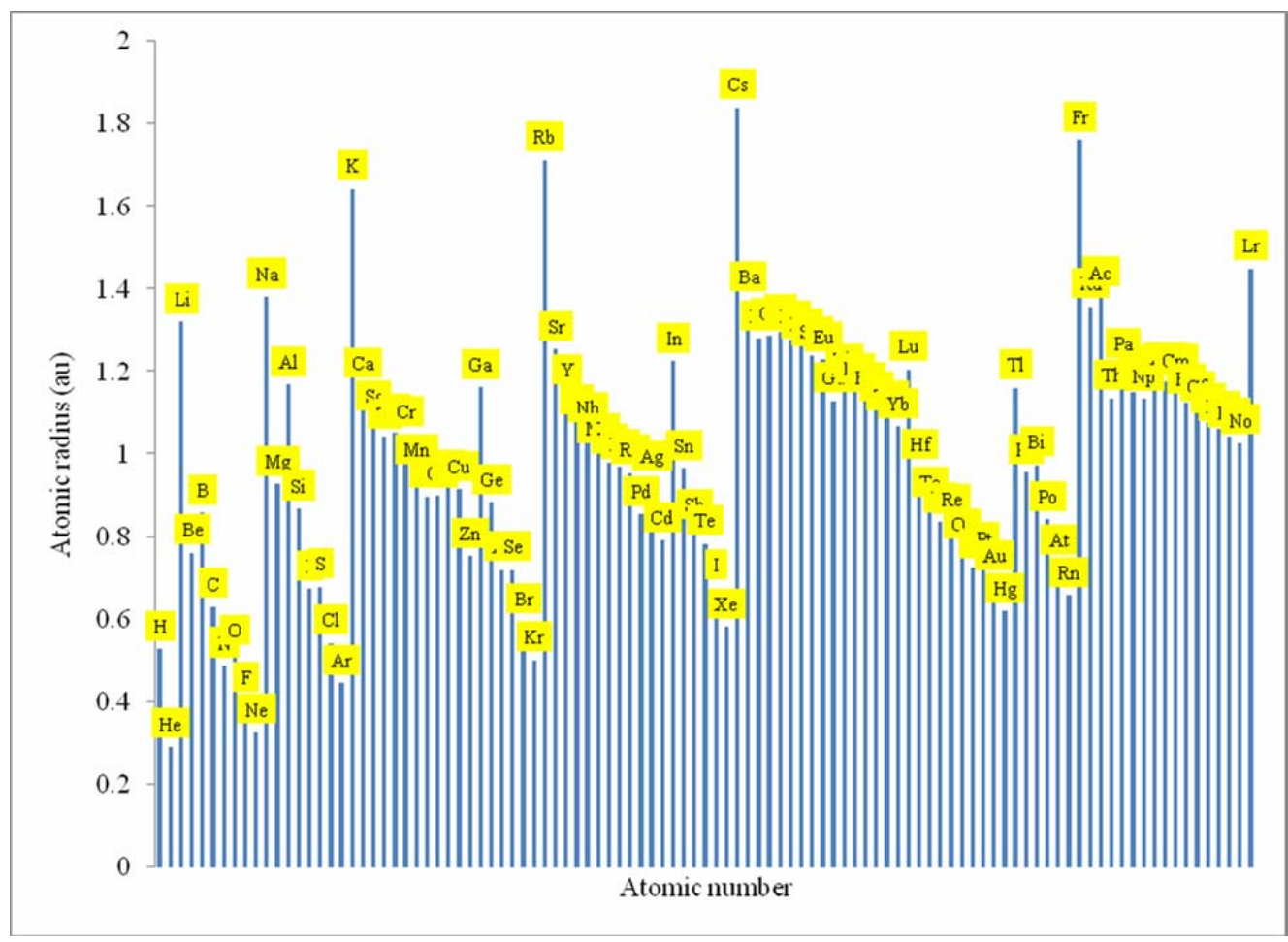

Fig. (1). Plot of computed atomic radii of 103 elements of the periodic table as a function of their atomic number.

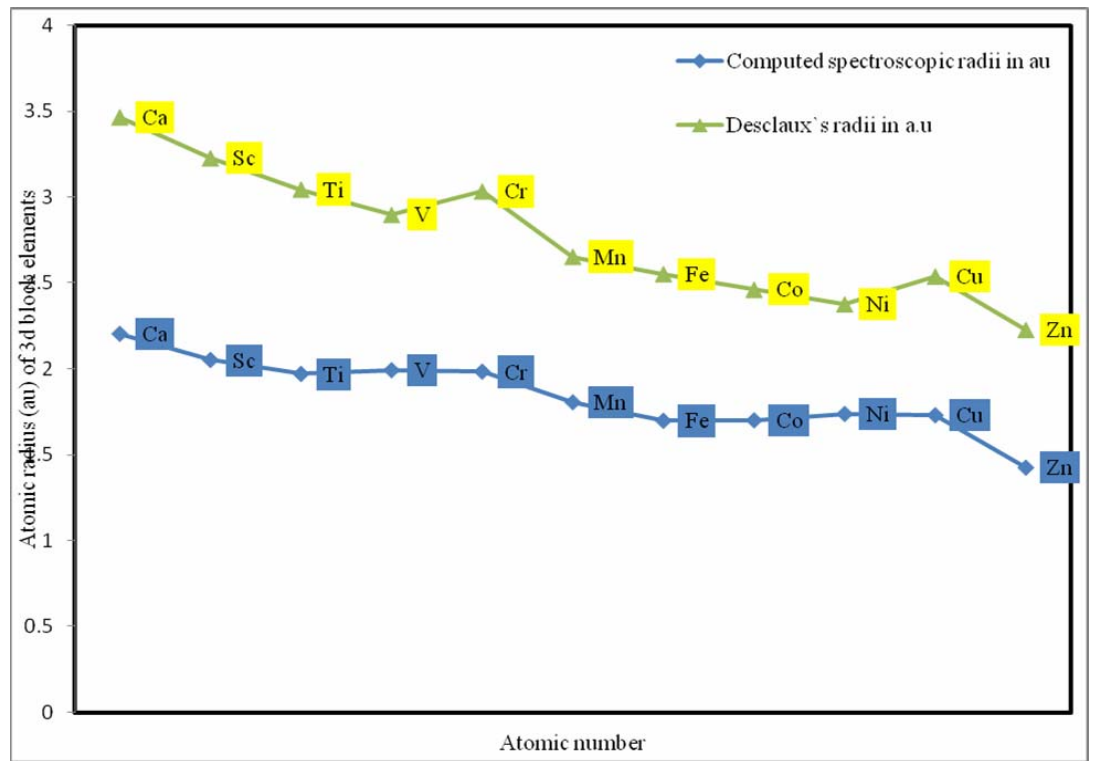

Fig. (2). Comparative plot of the computed atomic sizes vis-à-vis those computed by Desclaux of the 3d- bolck elements as a function of atomic number.

increased interest [55] over the past years in both experimental and theoretical studies because of their importance in astrophysical process and many chemical reactions.

\section{COMPUTATION OF EQUILIBRIUM INTER- NUCLEAR BOND DISTANCES OF HETERO NU- CLEAR DIATOMICS}

We have exploited the available ansatz for the evaluation of the inter nuclear distance of hetero nuclear diatomic molecules derived, on the basis of electronegativity equalization principle [38-40, 56-58], by Ray et al., [59]. We proceed to discuss the salient points of derivation of the necessary formula as follows-

Let us consider the formation of a diatomic molecule $\mathrm{AB}$ from its constituent atoms $\mathrm{A}$ and $\mathrm{B}$ as follows:

$\mathrm{A}+\mathrm{B} \rightarrow \mathrm{AB}$

Let the equilibrium bond length, the electronegativity of the molecule $\mathrm{AB}$, the charges and the electronegativities of the corresponding atoms, $\mathrm{A}$ and $\mathrm{B}$ are $\mathrm{R}_{\mathrm{AB}}, \chi_{\mathrm{AB}}, \mathrm{Z}_{\mathrm{A}}$ and $\mathrm{Z}_{\mathrm{B}}$, $\chi_{\mathrm{A}}$ and $\chi_{\mathrm{B}}$ respectively such that $\chi_{\mathrm{A}}>\chi_{\mathrm{B}}$. Now let us imagine that, after the formation of the molecule, a point charge, $\mathrm{q}$, is 


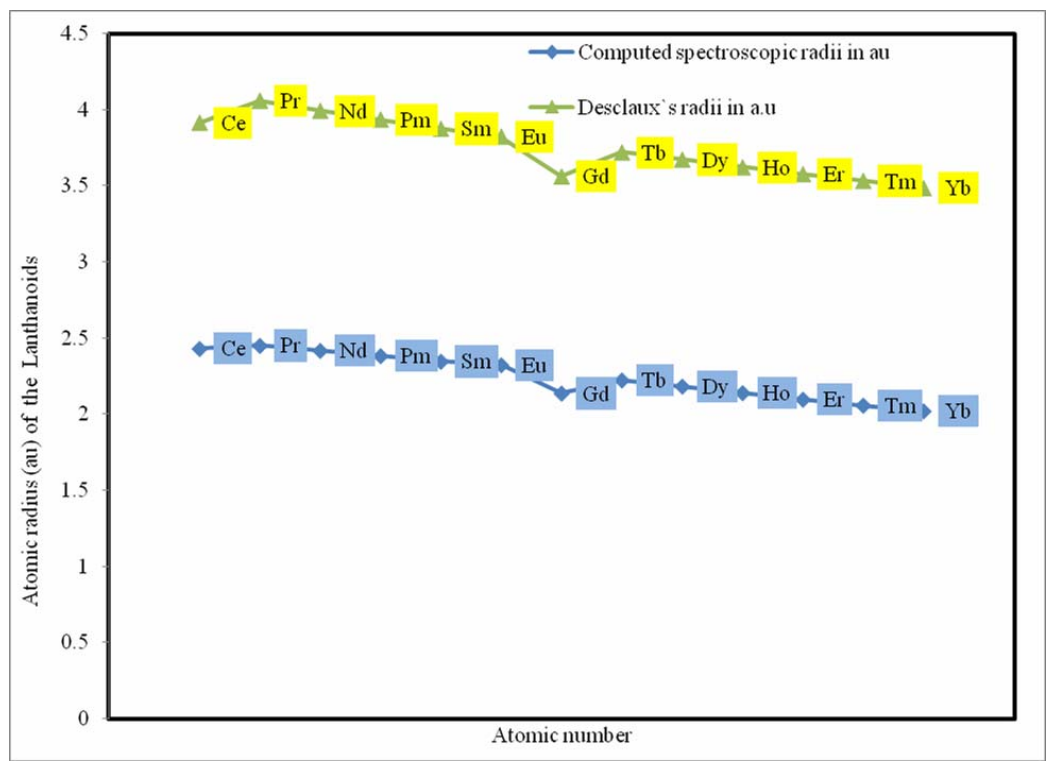

Fig. (3). Comparative plot of the computed atomic sizes vis-à-vis those computed by Desclaux of the Lanthanoids as a function of atomic number.

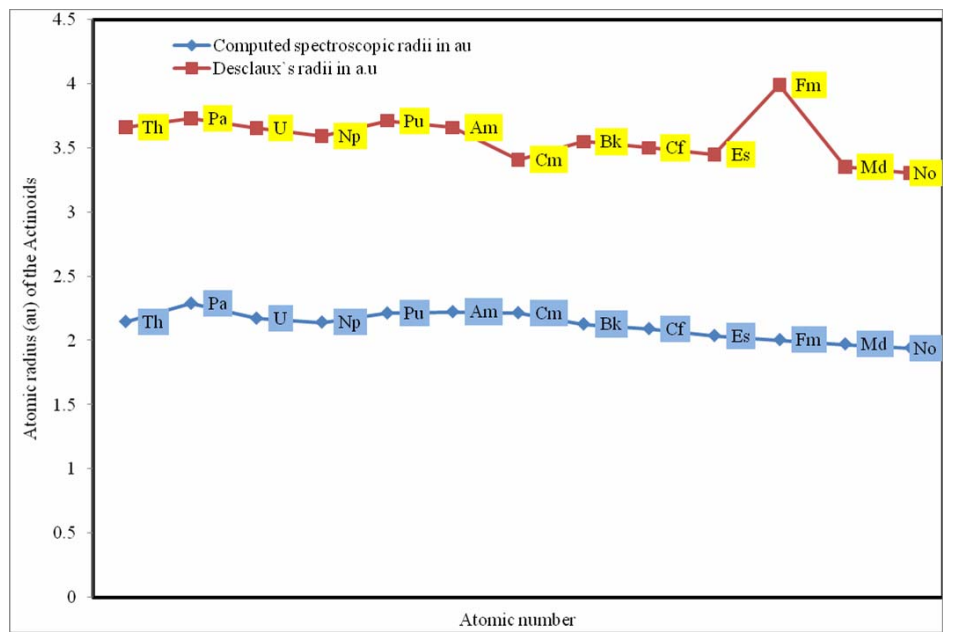

Fig. (4). Comparative plot of the computed atomic sizes vis-à-vis those computed by Desclaux of the Actinoids as a function of atomic number

located at a distance $\mathrm{r}_{1}$ from $\mathrm{A}$ and $\mathrm{r}_{2}$ from $\mathrm{B}$ with the condition that $r_{1}+r_{2}=R_{A B}$. In SBC [60-63] model, the charges of nuclei $A$ and $B$ in the molecule $A B$ are $Z_{A}+q$ and $Z_{B}-q$ respectively.

The distances $r_{1}$ and $r_{2}$ have been identified with the covalent radii of atoms $r_{A}$ and $r_{B}$ respectively (vide infra). Pasternak [64] suggested an ansatz for computing the electronegativity, $\chi$ of an atom as

$\chi=\mathrm{C} \mathrm{Z} / \mathrm{r}$

where $\mathrm{C}$ is a constant depending upon bond type, $\mathrm{Z}$ is the nuclear charge and $\mathrm{r}$ is the covalent radius of the atom.

When atoms approach to form the molecule the question as to what distance of separation between atoms do the chemical potential equalize is very much fundamental [65]. However, when atoms approach to form the molecule, the electron density function over the whole space undergoes rearrangement. Thus, during the chemical event of the formation of the molecule, there occurs a physical process of inter atomic charge transfer and rearrangement in hetero nu- clear molecules under the driving force of electronegativity equalization. Let the electronegativities of the isolated atoms $\mathrm{A}$ and $\mathrm{B}$ in the molecule $\mathrm{AB}$ are $\chi_{\mathrm{A}}^{\prime}$ and $\chi_{\mathrm{B}}^{\prime}$ respectively. The equilibrium inter nuclear distance we want to evaluate is $\mathrm{R}_{\mathrm{AB}}$. The electronegativity equalization principle of Sanderson [66] provides that the molecular electronegativity, $\chi_{\mathrm{AB}}$ has the following identity.

$\chi_{\mathrm{AB}}=\chi_{\mathrm{A}}^{\prime}=\chi_{\mathrm{B}}^{\prime}$

Now, on the basis of simple bond charge model [60-63], Ray et al., [59] derived the inter nuclear bond distances of diatomic molecules using the concept of electronegativity equalization $[59,66,67]$ and the zero order approximation of Pasternak [64] that $r_{A}=r_{1}$ and $r_{B}=r_{2}$.

After the charge transfer, the electronegativities of atoms $\mathrm{A}$ and $\mathrm{B}$ in the molecule $\mathrm{AB}$ according to the ansatz (27) are

$\chi_{\mathrm{A}}^{\prime}=\mathrm{C}\left(\mathrm{Z}_{\mathrm{A}}+\mathrm{q}\right) / \mathrm{r}_{1}$

and

$\chi_{\mathrm{B}}^{\prime}=\mathrm{C}\left(\mathrm{Z}_{\mathrm{B}}-\mathrm{q}\right) / \mathrm{r}_{2}$ 
and using the electronegativity equalization principle they obtained

$\chi_{\mathrm{AB}}=\mathrm{C}\left(\mathrm{Z}_{\mathrm{A}}+\mathrm{q}\right) / \mathrm{r}_{1}=\mathrm{C}\left(\mathrm{Z}_{\mathrm{B}}-\mathrm{q}\right) / \mathrm{r}_{2}$

After some trivial algebraic manipulation we have

$\chi_{\mathrm{AB}}=\left(\chi_{\mathrm{A}} \mathrm{r}_{\mathrm{A}}+\chi_{\mathrm{B}} \mathrm{r}_{\mathrm{B}}\right) /\left(\mathrm{r}_{1}+\mathrm{r}_{2}\right)$

Now putting $r_{A=1 / 2} R_{A A}$, and $r_{B}=1 / 2 R_{B B}$, and $R_{A B}=\left(r_{1}+r_{2}\right)$ in the Eq. (33) Roy et al., [59] obtained

$\chi_{\mathrm{AB}}=\left(\chi_{\mathrm{A}} \mathrm{R}_{\mathrm{AA}}+\chi_{\mathrm{B}} \mathrm{R}_{\mathrm{BB}}\right) / 2 \mathrm{R}_{\mathrm{AB}}$

Now, using SBC model for vibrational energy function and applying the hetero polar models for hetero nuclear diatomic molecules and homo polar models for homo nuclear diatomic molecules, Roy et al., [59] obtained

$\mathrm{R}_{\mathrm{AB}}=\left(\mathrm{r}_{\mathrm{A}}+\mathrm{r}_{\mathrm{B}}\right)-\left[\left\{\left(\mathrm{r}_{\mathrm{A}} \mathrm{r}_{\mathrm{B}}\left(\chi_{\mathrm{A}}{ }^{1 / 2}-\chi_{\mathrm{B}}{ }^{1 / 2}\right)^{2}\right\} /\left(\chi_{\mathrm{A}} \mathrm{r}_{\mathrm{A}}+\chi_{\mathrm{B}} \mathrm{r}_{\mathrm{B}}\right)\right]\right.$

where $r_{A}$ and $r_{B}$ are the covalent radii of atoms and $R_{A B}$ is the inter nuclear bond distance of the molecule, $\mathrm{AB}$.

We have made some amendment of the formula of Ray at al [59] by substituting the covalent radii $r_{A}$ and $r_{B}$ by their absolute radii, $\mathrm{r}_{\mathrm{A}}$ and $\mathrm{r}_{\mathrm{B}}$ in view of the fact the atoms remain grossly undistorted in molecules [68-70].

$\mathrm{R}_{\mathrm{AB}}=\left(\mathrm{r}_{\mathrm{A}}^{\prime}+\mathrm{r}_{\mathrm{B}}^{\prime}\right)-\left[\left\{\left(\mathrm{r}_{\mathrm{A}}^{\prime} \mathrm{r}_{\mathrm{B}}^{\prime}\left(\chi_{\mathrm{A}}^{1 / 2}-\chi_{\mathrm{B}}{ }^{1 / 2}\right)^{2}\right\} /\left(\chi_{\mathrm{A}} \mathrm{r}_{\mathrm{A}}^{\prime}+\chi_{\mathrm{B}} \mathrm{r}_{\mathrm{B}}^{\prime}\right)\right]\right.$

The equilibrium inter nuclear bond distance of as many as nine different sets of hetero nuclear diatomic are computed using the Eq. (36), the computed radii data of corresponding atoms $\mathrm{A}$ and $\mathrm{B}$, and the electronegativity values of those atoms published by us [15]. The calculated inter nuclear bond distances of the hetero nuclear diatomics vis-à-vis their experimental counterparts [71] are present in Table 2. The linear correlation coefficients $\left(R^{2}\right)$ of each set of compounds are also reported in the same table.

\section{RESULT AND DISCUSSION}

\section{Periodic Behaviour}

From Fig. (1) it is transparent that the computed absolute radii reproduce the periodicity of the periods and groups of periodic table perfectly. Each period begins with a representative element and absolute radii decreases monotonically horizontally to be minimum at the noble gas atoms that occur

Table 2. Comparative Study of the Evaluated Inter Nuclear Bond Distances of Nine Sets of Hetero Nuclear Diatomics vis-à-vis their Spectroscopic Counterparts and the Corresponding Square of the Correlation Constants

\begin{tabular}{|c|c|c|c|c|}
\hline \multirow[t]{2}{*}{ Sets } & \multirow[t]{2}{*}{ Molecule } & \multirow{2}{*}{$\begin{array}{r}\text { R(A-B) in Á } \\
\text { (This work) }\end{array}$} & \multirow{2}{*}{$\begin{array}{l}\mathbf{R}(\mathrm{A}-\mathrm{B}) \text { in } \AA \\
\text { (Experimental) }\end{array}$} & \multirow[t]{2}{*}{$\mathbf{R}^{2}$ value } \\
\hline & & & & \\
\hline \multirow[t]{16}{*}{ Alkali Halides: Set- I } & $\mathrm{LiF}$ & 1.56 & 1.56 & \multirow[t]{16}{*}{0.949} \\
\hline & $\mathrm{LiCl}$ & 1.78 & 2.02 & \\
\hline & $\mathrm{LiBr}$ & 1.82 & 2.02 & \\
\hline & LiI & 1.91 & 2.39 & \\
\hline & $\mathrm{NaF}$ & 1.62 & 1.93 & \\
\hline & $\mathrm{NaCl}$ & 1.84 & 2.36 & \\
\hline & $\mathrm{NaBr}$ & 1.89 & 2.5 & \\
\hline & $\mathrm{NaI}$ & 1.97 & 2.39 & \\
\hline & $\mathrm{KF}$ & 1.85 & 2.17 & \\
\hline & $\mathrm{KCl}$ & 2.08 & 2.67 & \\
\hline & $\mathrm{KBr}$ & 2.13 & 2.82 & \\
\hline & KI & 2.22 & 3.05 & \\
\hline & $\mathrm{RbF}$ & 1.89 & 2.27 & \\
\hline & $\mathrm{RbCl}$ & 2.13 & 2.79 & \\
\hline & $\mathrm{RbBr}$ & 2.17 & 2.94 & \\
\hline & $\mathrm{RbI}$ & 2.26 & 3.18 & \\
\hline \multirow[t]{4}{*}{ Hydrogen Halides: Set-II } & $\mathrm{HF}$ & 0.93 & 0.92 & 0.999 \\
\hline & $\mathrm{HCl}$ & 1.07 & 1.27 & \\
\hline & $\mathrm{HBr}$ & 1.12 & 1.41 & \\
\hline & $\mathrm{HI}$ & 1.2 & 1.61 & \\
\hline
\end{tabular}


Table 2. contd....

\begin{tabular}{|c|c|c|c|c|}
\hline \multirow[t]{2}{*}{ Sets } & \multirow[t]{2}{*}{ Molecule } & \multirow{2}{*}{$\begin{array}{r}\text { R(A-B) in Á } \\
\text { (This work) }\end{array}$} & \multirow{2}{*}{$\begin{array}{l}\mathbf{R}(\mathrm{A}-\mathrm{B}) \text { in } \AA \mathbf{A} \\
\text { (Experimental) }\end{array}$} & \multirow[t]{2}{*}{$\mathbf{R}^{2}$ value } \\
\hline & & & & \\
\hline \multirow[t]{6}{*}{ Inter Halogens: Set-III } & $\mathrm{ClF}$ & 0.94 & 1.63 & 0.967 \\
\hline & $\mathrm{BrF}$ & 0.99 & 1.76 & \\
\hline & $\mathrm{IF}$ & 1.06 & 1.91 & \\
\hline & $\mathrm{BrCl}$ & 1.14 & 2.14 & \\
\hline & $\mathrm{ICl}$ & 1.22 & 2.32 & \\
\hline & $\mathrm{IBr}$ & 1.22 & 2.48 & \\
\hline \multirow[t]{4}{*}{ Silver Halides: Set-IV } & $\mathrm{AgF}$ & 1.27 & 1.98 & 0.996 \\
\hline & $\mathrm{AgCl}$ & 1.46 & 2.28 & \\
\hline & $\mathrm{AgBr}$ & 1.51 & 2.39 & \\
\hline & $\mathrm{AgI}$ & 1.59 & 2.54 & \\
\hline \multirow[t]{6}{*}{ Binary Oxides: Set-V } & $\mathrm{CO}$ & 1.14 & 1.13 & 0.772 \\
\hline & NO & 1 & 1.15 & \\
\hline & $\mathrm{PbO}$ & 1.3 & 1.92 & \\
\hline & $\mathrm{BaO}$ & 1.43 & 1.94 & \\
\hline & $\mathrm{GeO}$ & 1.38 & 1.62 & \\
\hline & $\mathrm{SnO}$ & 1.45 & 1.83 & \\
\hline \multirow[t]{3}{*}{ Chalcogenides of $\mathrm{Pb}$ : Set-VI } & $\mathrm{PbS}$ & 1.54 & 2.29 & 0.994 \\
\hline & $\mathrm{PbSe}$ & 1.57 & 2.4 & \\
\hline & $\mathrm{PbTe}$ & 1.64 & 2.6 & \\
\hline \multirow[t]{3}{*}{ Chalcogenides of Ge: Set-VII } & GeS & 1.71 & 2.01 & 0.979 \\
\hline & $\mathrm{GeSe}$ & 1.77 & 2.13 & \\
\hline & GeTe & 1.82 & 2.34 & \\
\hline \multirow[t]{4}{*}{ Aluminum halides: Set-VIII } & $\mathrm{AlF}$ & 1.5 & 1.65 & 0.997 \\
\hline & $\mathrm{AlCl}$ & 1.69 & 2.13 & \\
\hline & $\mathrm{AlBr}$ & 1.74 & 2.29 & \\
\hline & AlI & 1.82 & 2.54 & \\
\hline \multirow[t]{4}{*}{ Thallium halides: Set-IX } & $\mathrm{TlF}$ & 1.32 & 2.08 & 0.991 \\
\hline & $\mathrm{TlCl}$ & 1.55 & 2.48 & \\
\hline & $\mathrm{TlBr}$ & 1.6 & 2.62 & \\
\hline & TII & 1.68 & 2.81 & \\
\hline
\end{tabular}

at the bottom of the curve. It is very interesting to note that the absolute size of nitrogen atom (0.92239 au) of the present work is smaller than the atomic radii of oxygen atom $(0.97822 \mathrm{au})$. The half shell stability of nitrogen atom is nicely reflected in present calculation of size. Since the atomic radius is neither a defined physical property nor it is constant under all circumstances, the scientific validity test of a set evaluated size data may be performed by observing the well recognized behavior of the atomic radii in the domain of chemical elements. These are:-
(1) Whether d- block contraction is manifest?

(2) Whether f- block contraction is manifest?

(3) Whether the influence of relativity, an effect finding its way into the present day chemistry of the elements [29-72-76], is manifest and evident in the size data?

(4) Whether peculiar physical and chemical behaviour of $\mathrm{Hg}$ and $\mathrm{Au}$ can be correlated in terms of evaluated size data? 
We shall try to examine whether the above four aspects are distinctly manifest in the size data evaluated by us. It is pertinent to mention that the Desclaux [29] calculation is perhaps the most sophisticated relativistic calculation of atomic size. We, therefore, heavily rely upon the size data of Desclaux [29] to set a standard or bench mark.

\section{The d-Block Contraction}

It is well known that the contraction of size due to screening effect [77] and relativistic effect in d-block elements are comparable [71-76]. We have extrapolated the sizes of the $3 \mathrm{~d}$ block elements of relativistic calculation of Desclaux [29] and that of present calculation in Fig. (2).

A look on the Fig. (2) reveals that the computed radii data of $3 \mathrm{~d}$ block elements correlates nicely with the data published by Desclaux [29]. A deeper look on the Fig. (2) further reveals that there is a steady decrease in radii in both set of data.

\section{The f-Block Contraction}

It is well known in chemistry that the sizes of the atoms and ions of lanthanide series are smaller than the expected. There is a regular decrease in their atomic and ionic radii and there is a regular decrease in their tendency to act as a reducing agent, with increase in atomic number. It is also opined, in general, that such decrease in size is due to the effect that results from the poor shielding of $4 \mathrm{f}$ electrons. But it has been argued vigorously by a number of workers [71-73] that decrease in size of lanthanide is not only due to poor shielding by $4 \mathrm{f}$ electrons but also due to effect of relativity. Relativity contracts s orbital strongly in preference to $\mathrm{p}, \mathrm{d}$, f orbitals. The effect of the lanthanide contraction is noticeable up to platinum $(Z=78)$, after which it is masked by a relativistic effect known as the inert pair effect.

However, it is estimated that the conjoint operation of shielding and relativity contracts the sizes of atoms and ions of lanthanides and post lanthanide elements. The estimated relativistic effect is about $10-15 \%$ [71-76]. It is the general conclusion that the lanthanide contraction is transmitted into the sizes and the physico- chemical properties of the post lanthanide elements.

We have extrapolated the sizes of the lanthanides and Actinoids of relativistic calculation of Desclaux [29] and that of present calculation in Figs. 3 and 4) respectively. A look on the Figs. (3 and 4) reveals that the Lanthanoid contraction and Actinoide contraction is present in both sets of data- the present calculation and the data published by Desclaux [29]. Thus the chemico-physical behaviour of Lanthanoids and Actinoids can nicely be correlated by the size data of present calculation.

The color of the gold atom and liquid state of $\mathrm{Hg}$ at ambient temperature and its inertness are correlated to the relativistic effect, which make their sizes very small.

The evaluated radii of $\mathrm{Hg}(1.1727 \mathrm{au})$ and $\mathrm{Au}$ (1.319757 au) are very small. The peculiar chemico-physical behaviour of $\mathrm{Hg}$ is correlated to its small size. We [24] have already pointed out that, for a series of lanthanide (III) ions, the ex- perimental ionic radii [78] decreases steadily. It is expected that the lanthanoids are bigger than actinoids [79]. A closer look into Table $\mathbf{1}$ and Fig. (1) reveals that this trend is visibly distinct in our computed size data.

\section{Application of the Theoretically Calculated Absolute Ra- dii of Atoms in the Real World}

In this report, we have applied our absolute radii values of the atoms to compute the equilibrium inter nuclear distances of nine sets of widely divergent hetero nuclear diatomics. The evaluated inter nuclear bond distances of the molecules under reference through the formula (36) and their experimental counterparts are reported in the Table 2. A close look in to the Table 2 reveals that there is a very good correlation between the inter nuclear bond distances of the nine sets of molecules computed through ansatz (36) using the computed size data and the electronegativity values published by us [15] and their spectroscopically evaluated counter parts. It is surprising to note that the linear correlation coefficient value $\left(\mathrm{R}^{2}\right)$ of each set of compound is above 0.9 . The only apparent deviation we have noted is with some double boned species. The linear correlation coefficient value $\left(R^{2}\right)$ of this set also deviates much from the others. The apparent deviation can be attributed to the inherent limitation of the model to calculate inter nuclear bond distance. The ansatz (36) is derived for single bonded covalent compounds and the covalent radii are substituted by their absolute radii in view of the fact the atoms remain grossly undistorted in molecules. But for multiply bonded compounds, the model is bound to fail. This is evident in the data Set $-\mathrm{V}$ in Table 2 the correlation between theoretically computed inter-nuclear bond distance and experimental bond distance of multiply bonded compounds have poor correlation. However, the dictum -theory predicts and experiment proves is satisfied in this work.

\section{CONCLUSION}

In this venture we have evaluated a new set absolute radii of 103 elements of the periodic table in terms of the atomic spectroscopic data. We have linked the concept of hardness and the simple Bohr model of atomic structure. The estimated set of radii appears to satisfy the entire 'sine qua non' of sizes of atoms of the periodic table. Relativistic effect appears to have significantly included in the suggested algorithm evaluating the atomic radii. We have found that the sizes of $\mathrm{Hg}$ and $\mathrm{Au}$ and other post lanthanide elements in our calculation are consistent with their physico-chemical properties. Since there is no experimental or theoretical benchmark to perform a validity test of any set of atomic size, we have connected our size data in the real world. We have calculated the real world descriptor like equilibrium inter nuclear distances of a good number of hetero nuclear diatomic molecules in terms of our computed absolute radii of atoms and compared with their experimental counter parts. The express good correlation between the set of theoretically evaluated inter nuclear distance and their experimental counter parts, and the explicit adherence to the well known physico-chemical behaviour of atoms by the present set of radii strongly suggest that the present method of calculating atomic radii is a meaningful venture. 


\section{REFERENCES}

[1] Feynman RP, Leighton RB, Sands M. The feynman lecture on physics. Boston: Addison- Wesley 1964; vol. 1.

[2] Ghosh DC. A new scale of electronegativity based on absolute radii of atoms. J Theor Comput Chem 2005; 4: 21-33

[3] Ghosh DC, Biswas R. Theoretical calculation of absolute radii of atoms and ions. part 1. the atomic radii. Int J Mol Sci 2002; 3: 87113.

[4] Ghosh DC, Islam N. Semiempirical evaluation of the global hardness of the atoms of 103 elements of the periodic table using the most probable radii as their size descriptors. Int J Quantum Chem 2009; 110: 1206-13.

[5] Bohr N. On the constitution of atoms and molecules. Philos Mag 1913; 26: 1-25.

[6] Saha M. Ionisation in the solar chromosphere. Nature 1920; 105: 232-3.

[7] Basu SN. On the deduction of Rydberg's law from the quantum theory of spectral emission. Phil Mag Sci 1920; 40: 619-27.

[8] Eve AS. Ionisation potential and the size of the atom. Nature 1921; 107: 552-3.

[9] Tamura S. An empirical correlation between the atomic radius and the first ionization energy for elements. J Mater Sci Lett 1996; 15: $1678-81$

[10] Bohórquez HJ, Boyd R J. Is the size of an atom determined by its ionization energy? Chem Phys Lett 2009; 480: 127-31.

[11] Sabin JR, Trickey SB, Apell SP, Oddershede J. molecular shape, capacitance, and chemical hardness. Int J Quantum Chem 2000; 77: 358-66.

[12] Islam N, Ghosh DC. A New radial dependent electrostatic algorithm for the evaluation of the electrophilicity indices of the atoms. Int J Quantum Chem 2010; DOI: 10.1002/qua.22861: (online).

[13] Frenking G, Krapp A. Unicorns in the world of chemical bonding models. J Comput Chem 2007; 28: 15-24.

[14] Ayers PW. The physical basis of the hard/soft acid/base principle. Faraday Discuss 2007; 135: 161-90.

[15] Ghosh DC, Islam N. whether electronegativity and hardness are manifest two different descriptors of the one and the same fundamental property of atoms -a quest. Int J Quantum Chem 2011; 111: 40-51.

[16] Bragg WL. Hypothesis of constant atomic radii. Phil Mag J Sci 1920; 40: 169-83.

[17] Slater JC. Atomic shielding constants. Phys Rev 1930; 36: 57-64.

[18] Hartree DR. calculation of atomic structures. New York: Wiley 1957.

[19] Clementi E, Raimondi DL. atomic screening constants from scf functions. J Chem Phys 1963; 38: 2686-9.

[20] Yang Z-Z, Davidson ER. Evaluation of a characteristic atomic radius by an ab initio method. Int J Quantum Chem 1997; 62: 4753.

[21] Waber JT, Cromer DT. Orbital radii of atoms and ions. J Chem Phys 1965; 42: 4116-23.

[22] Froese C. Hartree-Fock parameters for the atoms helium to radon. J Chem Phys 1966; 45: 1417-20.

[23] Clementi E, Raimondi DL, Reinhardt WP. atomic screening constants from scf functions. II. Atoms with 37 to 86 electrons. J Chem Phys 1967; 47: 1300-07.

[24] Larson AC, Waber JT. Self-consistent field hartree calculations for atoms and ions. Report LA-4297, LOS Alamos: QSAEC 1969.

[25] Fischer CF. Average-energy-of-configuration Hartree-Fock results for the atoms helium to radon. Atom Data Nucl Data Tables 1972; 4: 301-99.

[26] Kammeyer CW, Whitman DR. Quantum Mechanical calculation of molecular radii. i. hydrides of elements of periodic groups IV through VII. J Chem Phys 1972; 56: 4419-21.

[27] Fraga S, Karwoueski J, Saxena KMS. Hartree-Fock values of coupling constants, polarizabilities, susceptibilities, and radii for the neutral atoms, helium to nobelium. Atom Data Nucl Data Tables 1973; 12: 467-77.

[28] Fischer CF. Average-energy-of-configuration Hartree-Fock results for the atoms helium to radon Atom Data Nucl Data Tables 1973; 12: 87-99.

[29] Desclaux JP. Relativistic Dirac-Fock expectation values for atoms with $Z=1$ to $Z=120$. Atom Data Nucl Data Tables 1973; 12: 311-406.

[30] Boyd RJ. The relative sizes of atoms. J Phys B Atom Molec Phys 1977; 10: 2283-95.
[31] Deb B M, Singh R, Sukumar N. A universal density criterion for correlating the radii and other properties of atoms and ions. J Mol Struct (Theochem) 1992; 259: 121-39.

[32] Nath S, Bhattacharya S, Chattaraj PK. Density functional calculation of a characteristic atomic radius. J Mol Struct (Theochem) 1995; 331: 267-79.

[33] Putz MV, Russo N, Sicilia E. Atomic radii scale and related size properties from density functional electronegativity formulation. $\mathrm{J}$ Phys Chem A 2003; 107: 5461-5.

[34] Pyykkö P, Riedel S, Patzschke M. Triple-bond covalent radii. Eur J Chem 2005; 11: 3511-20.

[35] Pyykkö P, Atsumi M. Molecular single-bond covalent radii for elements 1-118. Eur J Chem 2008; 15: 186-97.

[36] Ghosh DC, Biswas R, Chakraborty T, Islam N, Rajak SK. The wave mechanical evaluation of the absolute radii of atoms. J Mol Struct (Theochem) 2008; 865: 60-7.

[37] Chakraborty T, Gazi K, Ghosh DC. Computation of the atomic radii through the conjoint action of the effective nuclear charge and the ionization energy. Mol Phys 2010; 108: 2081-92.

[38] Ghosh DC, Islam N. Whether there is a Hardness Equalization Principle Analogous to the Electronegativity Equalization PrincipleA Quest. Int J Quantum Chem 2010; DOI 10.1002/qua.22508,online.

[39] Ghosh DC, Islam N. A quest for the algorithm for evaluating the molecular hardness. Int $\mathrm{J}$ Quantum Chem 2010; DOI 10.1002/qua.22499, online.

[40] Ghosh DC, Islam N. Charge transfer associated with the physical process of hardness equalization and the chemical event of the molecule formation and the dipole moments Int. J Quantum Chem 2010; DOI 10.1002/qua.22653 online.

[41] Islam N, Ghosh DC. A new algorithm for the evaluation of equilibrium internuclear bond distance of heteronuclear diatomic molecule based on the hardness equalization principle. Eur Phys J D 2011; DOI: 10.1140/epjd/e2010-10058-6 online.

[42] Hohenberg P, Kohn H. Inhomogeneous electron gas. Phys Rev 1964; 136: 864-71.

[43] Parr RG, Yang W. Density functional theory of atoms and molecules. New York: Oxford University Press 1989.

[44] Gyftpoulous EP, Hatsopoulos GN. Quantum-thermodynamic definition of electronegativity. Proc Natl Acad Sci 1968; 60: 786-93.

[45] Iczkowski RP, Margrave JL. Electronegativity. J Am Chem Soc 1961; 83: 3547-51.

[46] Parr RG, Donnelly RA, Levy M, Palke WE. Electronegativity: the density functional viewpoint. J Chem Phys 1978; 68: 3801-7.

[47] Parr RG, Pearson RG. Absolute hardness: companion parameter to absolute electronegativity. J Am Chem Soc 1983; 105: 7512-6.

[48] Pearson RG. Absolute electronegativity and hardness correlated with molecular orbital theory. Proc Natl Acad Sci 1986; 83: 8440-1

[49] Perdew JP, Parr RG, Levy M, Balduz Jr JL. density-functional theory for fractional particle number: derivative discontinuities of the energy. Phys Rev Lett 1982; 49: 1691-4.

[50] Yang WT, Zhang YK, Ayers PW. degenerate ground states and a fractional number of electrons in density and reduced density matrix functional theory. Phys Rev Lett 2000; 84: 5172-5.

[51] Sen KD, Vinayagam SC. Absolute hardness parameter: finite difference versus density functional theoretic definition. Chem Phys Let $1988 ; 144: 178-9$.

[52] Komorowski L. Electronegativity and hardness in the chemical approximation. Chem Phys 1987; 114: 55-71.

[53] Rienstra-Kiracofe JC, Tschumper GS, Schaefer III HF. atomic and molecular electron affinities: photoelectron experiments and theoretical computations. Chem Rev 2002; 102: 231-82.

[54] Sansonctti JE, Martin WC. Handbook of basic atomic spectroscopic data. USA: American institute of physics 2005

[55] Noorizadeh S, Pourshams GR. New empirical potential energy function for diatomic molecules. J Mol Struct (Theochem) 2004; 678: 207-10.

[56] Datta D. Geometric mean principle for hardness equalization: a corollary of Sanderson's geometric mean principle of electronegativity equalization. J Phys Chem 1986; 90: 4216-7.

[57] Berkowitz M, Ghosh SK, Parr RG. On the concept of local hardness in chemistry, J Am Chem Soc 1985; 107: 6811-20.

[58] Ghosh SK, Berkowitz M, Parr RG. Transcription of ground-state density-functional theory into a local thermodynamics Proc Natl Acad Sci 1984; 81: 8028-31

[59] Ray NK, Samuels L, Parr RG. Studies of electronegativity equalization. J Chem Phys 1979; 70: 3680-4. 
[60] Parr RG, Borkman RF. Chemical binding and potential-energy functions for molecules. J Chem Phys 1967; 46: 3683-5.

[61] Borkman RF, Parr RG. Toward an understanding of potential-energy functions for diatomic molecules. J Chem Phys 1968; 48: 1116-26.

[62] Parr RG, Borkman RF. Simple bond charge model for potentialenergy curves of homonuclear diatomic molecules. J Chem Phys 1968; 49: 1055-8.

[63] Borkman RF, Simons G, Parr RG. Simple bond-charge model for potential-energy curves of heteronuclear diatomic molecules. J Chem Phys 1969; 50: 58-63.

[64] Pasternak A. Electronegativity based on the simple bond charge model. Chem Phys 1977; 26: 101-12.

[65] Perdew JP, Parr RG, Levy M, Balduz JL. Density-functional theory for fractional particle number: derivative discontinuities of the energy. Phys Rev Lett 1982; 49: 1691-9.

[66] Sanderson RT, An interpretation of bond lengths and a classification of bonds. Science 1951; 114: 670-672.

[67] De Proft F, Langenaeker W, Geerlings P. A non-empirical electronegativity equalization scheme. Theory and applications using isolated atom properties. J Mole Struct(Theochem) 1995; 339: 45-55.

[68] Murphy LR, Meek TL, Allred AL, Allen LC. evaluation and test of pauling's electronegativity scale. J Phys Chem A 2000; 104: 5867-71.
[69] Nalewajski RF, Parr RG. Information theory, atoms in molecules, and molecular similarity. Proc Natl Acad Sci 2000; 97: 8879-82.

[70] Bader RFW. A quantum theory of molecular structure and its applications. Chem Rev 1991; 91: 893-928.

[71] Lovas FJ, Tiemann E. Microwave spectral tables i. diatomic molecules. Phys Chem Ref Data 1974; 3: 609-770.

[72] Desclaux JP, Ki-Kim Y. Relativistic effects in outer shells of heavy atoms. J Phys B Atom Mol Phys 1975; 8: 1177-89.

[73] Pitzer KS. Relativistic effects on chemical properties. Acc Chem Res 1979; 12: 271-6.

[74] Pyykkö P, Desclaux JP. Relativity and the periodic system of elements. Acc Chem Res 1979; 12: 276-81.

[75] Pyykkö P. Relativity and the periodic system of elements. Chem. Rev 1988; 88: 563-94.

[76] Norrby LJ. Why is mercury liquid? Or, why do relativistic effects not get into chemistry textbooks? J Chem Educ 1991; 68: 110-1.

[77] Lloyd DR. On the lanthanide and "scandinide" contractions. J Chem Educ 1986; 63: 502-4.

[78] Ahrens LH. The use of ionization potentials Part 1. Ionic radii of the elements. Geochim Cosmochim Acta 1952; 2: 155-69.

[79] Indelicato P, Santos JP, Boucard S, Desclaux JP. QED and relativistic corrections in superheavy elements. Eur Phys J D 2007; 45: 155-62.

(C) Islam and Ghosh; Licensee Bentham Open.

This is an open access article licensed under the terms of the Creative Commons Attribution Non-Commercial License (http://creativecommons.org/licenses/ by-nc/3.0/) which permits unrestricted, non-commercial use, distribution and reproduction in any medium, provided the work is properly cited. 